\title{
Mortality in the special care baby unit of the main children's hospital in Tripoli, Libyan Arab Jamahiriya
}

A.M.E. Abushhaiwia, ${ }^{1}$ M.M.N Ziyani' and M. Dekna ${ }^{7}$

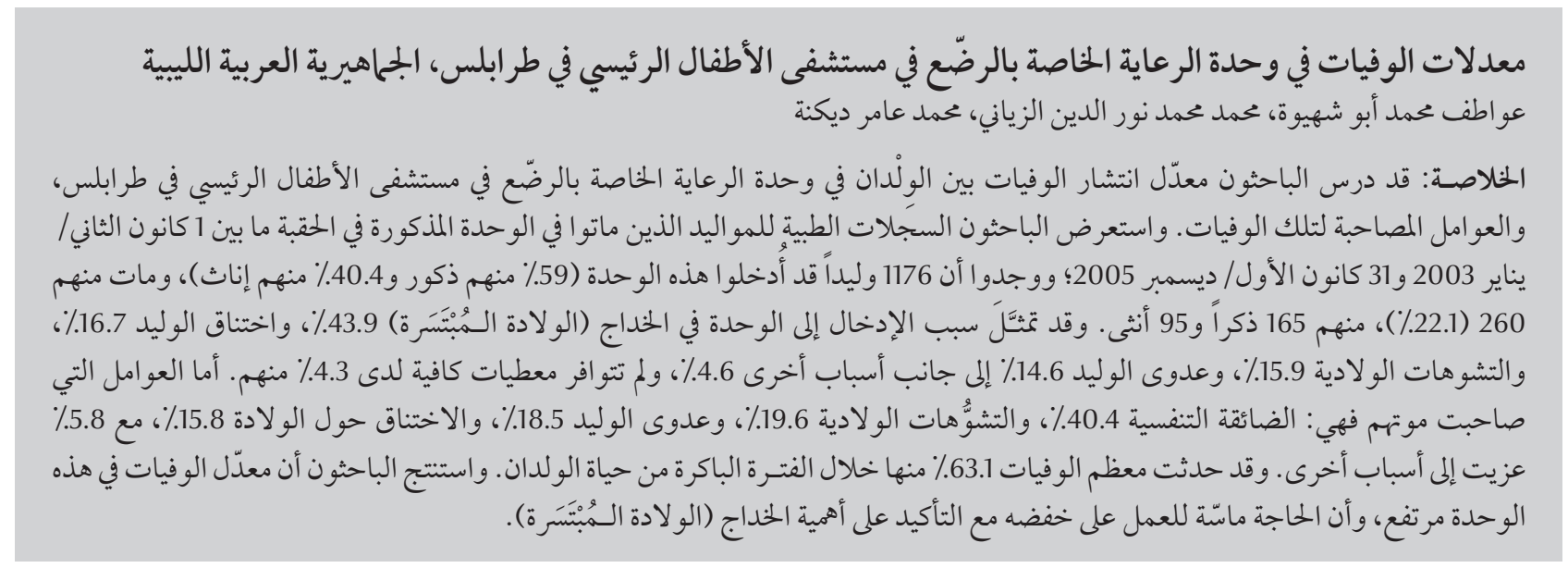

ABSTRACT We investigated the prevalence of neonatal deaths in the special care baby unit (SCBU) at the main children's hospital in Tripoli and the factors associated with these deaths. We reviewed the medical records of all neonates who died in our SCBU from 1 January 2003 to 31 December 2005. A total of 1176 neonates were admitted to our SCBU (59.6\% males and 40.4\% females). Of these, 260 (22.1\%) died: 165 males and 95 females. The reasons for admission to SCBU of the neonates who died were: prematurity $(43.9 \%)$, birth asphyxia $(16.7 \%)$, congenital malformations (15.9\%), neonatal infections (14.6\%), other reasons $(4.6 \%)$ and data unavailable $(4.3 \%)$. The factors associated with death were: respiratory distress $(40.4 \%)$, congenital malformation (19.6\%), neonatal infections (18.5\%) and perinatal asphyxia (15.8\%), with 5.8\% attributed to other causes. The majority of deaths (63.1\%) occurred during the early neonatal period. Mortality in our SCBU is high and action is needed to reduce it with emphasis on prematurity.

Mortalité dans l'unité néonatale de soins intensifs du principal hôpital d'enfants à Tripoli (Jamahiriya arabe libyenne)

RÉSUMÉ Nous avons étudié la prévalence des décès de nouveau-nés dans l'unité néonatale de soins intensifs du principal hôpital pour enfants de Tripoli et les facteurs associés à ces décès. Nous avons étudié les dossiers médicaux de tous les nouveau-nés qui y sont décédés entre le 1er janvier 2003 et le 31 décembre 2005. Au total, 1176 nouveau-nés ont été admis dans cette unité de soins (59,6\% de garçons et $40,4 \%$ de filles). Parmi eux, $260(22,1 \%)$ sont décédés : 165 garçons et 95 filles. Les raisons plus lesquelles les nouveau-nés décédés avaient été hospitalisés étaient les suivantes : prématurité $(43,9 \%)$, asphyxie néonatale (16,7\%), malformations congénitales $(15,9 \%)$, infections néonatales $(14.6 \%)$, autres raisons $(4,6 \%)$ et données non disponibles $(4,3 \%)$. Les facteurs associés aux décès étaient les suivants : détresse respiratoire (40,4\%), malformation congénitale $(19,6 \%)$, infections néonatales $(18,5 \%)$ et asphyxie périnatale $(15,8 \%)$, et 5,8 \% attribués à d'autres causes. La majorité des décès $(63,1 \%)$ est survenue pendant la période néonatale précoce. La mortalité dans cette unité de soins est élevée et une action est nécessaire pour la réduire, en mettant l'accent sur la prématurité.

'Tripoli Children's Hospital, Tripoli, Libyan Arab Jamahiriya (Correspondence to M. Dekna:drdekna@yahoo.com; A.M.E.Abushhaiwia: afma200595@hotmail.com;M.M.NZiyani:drziyani@hotmail.com).

Received: 12/03/09; accepted: 29/06/09 


\section{Introduction}

Both fetal and extra-uterine life form a continuum during which human growth and developmentare affected bygenetic, environmental and social factors [1]. The perinatal period is defined as the period extending from the 24th week of gestation to the end of the first week of life. Perinatal mortality includes both still births and early neonatal deaths [2]. The perinatal mortality rate is defined as number of still births and early neonatal deaths per 1000 total births.

A high perinatal mortality rate is an indicator of poor maternal health care, inappropriate management of delivery and inadequate newborn care. Babies die after birth because they have difficulty adapting to extra-uterine life, are born prematurely, are severely malformed, suffer from obstetric complications or because of unhygienic practices after birth that lead to infection [2].

The neonatal mortality rate is defined as number of neonatal deaths per 1000 live births. Across the globe neonatal mortality rates range from 5 per 1000 in developed countries to 50 per 1000 in the least developed countries; early neonatal mortality represents about $75 \%$ of neonatal mortality [2]. Globally, approximately 7.5 million infants die annually, more than half of these deaths occur during the neonatal period and $98 \%$ of the neonatal deaths occur in the developing regions [2].

Early neonatal deaths (occurring in the first 7 days of life) are the joint responsibility of obstetricians and paediatricians. Provision of adequate neonatal care remains a major challenge in countries with poor resources where the birth rate is high and health facilities are few and with poorly equipped special care baby units (SCBUs).

Neonatal medical services as a paediatric subspecialty was established on a wide scale in the Libyan Arab Jamahiriya in 1985. In 1990 the first report on morbidity and mortality rates in
Libyan neonates in Algala Children and Obstetrics Hospital was issued [3]. The perinatal mortality rate at that time was 26.3 per 1000 total births, which was high compared to western European and North American countries. The major causes of neonatal deaths in Tripoli during the early 1990s were premature birth and its complications, low birth weight, congenital malformations, infections, perinatal asphyxia and birth trauma.

The aim of the present study was to determine the factors associated with neonatal death in the SCBU of the Tripoli children's hospital. This is the main children's hospital in the western part of the Libyan Arab Jamahiriya.

\section{Methods}

The study carried out in the SCBU of Tripoli children's hospital, which provides medical services to paediatric age groups (birth to 16 years old), and admits sick infants referred from primary and secondary health institutions mainly over the western part of the country. The SCBU has a bed capacity of 20 beds and admits an average of 350 infants per year. The nurse/patient ratio is $1: 4$. Facilities are available for basic care. Laboratory facilities include complete blood count, blood sugar, renal function test, blood group and bilirubin, which are done as a routine. Infection screening for bacteria and sepsis work-up, e.g. blood and urine cultures, and spinal tap, are performed when newborns are suspected to be infected clinically.

Over a 3-year period (1 January 2003 to 31 December 2005), the medical records of all neonates who died in the SCBU were reviewed. Data were abstracted and filled in a predesigned worksheet. No autopsy was performed on any of the infants who died. For the purposes of this study we applied the definitions according to the WHO classifications of diseases [4].
SPSS software, version 10 was used to analyse the collected data. $P<0.05$ was considered statistically significant.

\section{Results}

Table 1 shows the annual admissions to the SCBU over the study period and the proportion that died. During the study period, 1176 babies were admitted to our SBCU, 260 of whom died (22.1\%). There was a slight drop in the number of admissions and a rise in mortality over the 3 years of the study.

Table 1 also shows that 357 babies were referred from Algala Maternity Hospital, $38.4 \%$ of whom died, 182 babies came from private clinics $(24.7 \%$ died), 220 from other hospitals (19.1\% died ) and 417 babies came directly from home ( $8.6 \%$ died).

As regards sex, 701 (59.6\%) babies admitted were males and 475 (40.4\%) females. This sex difference was statistically significant $(P<0.0001)$ (Table 1$)$. Of these, 165 male babies died (23.5\%) and 95 (20.0\%) female babies died, but the difference was not statistically significant $(P=0.051)$.

The reasons for admission to SCBU of the neonates who died were: prematurity (43.9\%), birth asphyxia (16.7\%), congenital malformations (15.9\%), neonatal infections (14.6\%) and other reasons (4.6\%).

The age at admission of the neonates that died ranged from 30 minutes to 28 days. We found that $84.5 \%$ of the babies that died were admitted during the early neonatal period and $15.5 \%$ were admitted during the late neonatal period (between 7th and 28th day of life), which means that although the early neonatal period is only one-fourth of the total neonatal period, more admissions occurred during this early period.

More than half $(56.1 \%)$ of the neonates who died were delivered by normal vaginal delivery. Emergency caesarean section was the mode of 


\begin{tabular}{|c|c|c|c|c|c|c|}
\hline \multirow[t]{2}{*}{ Variable } & \multicolumn{2}{|c|}{ Admissions } & \multirow[t]{2}{*}{$P$-value } & \multicolumn{2}{|c|}{ Deaths } & \multirow[t]{2}{*}{$P$-value } \\
\hline & No. & $\%$ & & No. & $\%$ & \\
\hline Year & & & 0.012 & & & 0.164 \\
\hline 2003 & 434 & 36.9 & & 83 & 19.1 & \\
\hline 2004 & 391 & 33.2 & & 87 & 22.3 & \\
\hline 2005 & 351 & 29.8 & & 90 & 25.6 & \\
\hline Referral place & & & 0.001 & & & 0.001 \\
\hline Algala Maternity Hospital & 357 & 30.4 & & 137 & 38.4 & \\
\hline Private clinics & 182 & 15.5 & & 45 & 24.7 & \\
\hline Other hospitals & 220 & 18.7 & & 42 & 19.1 & \\
\hline Self-referrals & 417 & 35.5 & & 36 & 8.6 & \\
\hline Sex & & & 0.001 & & & 0.051 \\
\hline Males & 701 & $59.6^{\mathrm{a}}$ & & 165 & $23.5^{\mathrm{b}}$ & \\
\hline Females & 475 & 40.4 & & 95 & 20.0 & \\
\hline Total & 1176 & 100.0 & & 260 & 22.1 & \\
\hline
\end{tabular}

delivery in $29.7 \%$ of the newborns who died and $7.9 \%$ were delivered by elective caesarean section. Instrumental delivery was used in $2.5 \%$ of the cases; data about delivery were unavailable $3.8 \%$ of the neonates who died.

The birth weight of the neonates who died in the SCBU during the study period ranged from $520 \mathrm{~g}$ to $4700 \mathrm{~g}$ with a mean of 2020 (standard deviation of 117) g: $40 \%$ were normal birth weight, $33 \%$ were low birth rate, $16 \%$ very low birth rate $(<1500 \mathrm{~g})[2,3], 10 \%$ extremely low birth weight $(<1000 \mathrm{~g})$ $[2,3]$ and only $1 \%$ were macrosomic.

Of the babies that died, $52.3 \%$ were born preterm, $44.8 \%$ were born at term and $1.7 \%$ were born post term, the difference between these three categories was statistically significant $(P<0.0001)$.
Data were unavailable for $1.2 \%$ of the neonates who died.

Of the 260 neonates who died, 164 (63.1\%) died during the early neonatal period, while 80 (30.8\%) died during late neonatal period and 16 babies (6.2\%) died during the post-neonatal period.

Table 2 shows that the most common factors associated with death were: respiratory distress $(105 ; 40.4 \%)$, congenital malformation $(51 ; 19.6 \%)$, infection $(48 ; 18.5 \%)$, perinatal asphyxia (41; 15.8\%). Other pathological problems such as birth trauma, severe hypertonic dehydration, kernicterus and intraventricular haemorrhage were factor in 15 (5.7\%) deaths.

Blood cultures were done for 119 neonates who died, $57 \%$ of which produced no growth (Table 3 ). The

\begin{tabular}{lcc}
\hline Table 2 Distribution of neonatal deaths according to condition suffered \\
Factors associated with death & No. & $\%$ \\
Respiratory distress & 105 & 40.4 \\
Congenital malformation & 51 & 19.6 \\
Infections & 48 & 18.5 \\
Birth asphyxia & 41 & 15.8 \\
Others & 15 & 5.8 \\
Total & 260 & 100.0 \\
\hline
\end{tabular}

predominant organisms found to have caused neonatal sepsis in our study were Klebsiella spp. (22.7\%), Staphylococcus aureus (13.5\%) and Pseudomonas aeruginosa $(3.3 \%)$.

\section{Discussion}

In our study the overall death rate was $22.1 \%$ among the neonates admitted to the SCBU of the Tripoli children's hospital during the study period; $63.1 \%$ of deaths occurred during the early neonatal period. These finding are in agreement with a study at the intensive care unit in Abha Saudi Arabia 22.4\% of admitted neonates died and $61 \%$ of deaths occurred within the first 3 days of life [5] (Table 4). Our figure is slightly lower than the $24.6 \%$ death rate reported from a study in Kenya [6] but slightly higher than the $18.0 \%$ death rate reported in an SCBU in Uganda [7]. It is higher than the $14.1 \%$ mortality reported in a study carried out in Taipei, Taiwan [5] and considerably higher than the SCBU death rate reported in Canada (4.1\%) [8] and the United States of America (0.8\%) [9]. This illustrates that while neonatal mortality in developed countries has fallen dramatically, developing 


\begin{tabular}{lcc}
\hline Table 3 Distribution of neonatal deaths according to blood culture results \\
\hline Type of organism & No. & $\%$ \\
No growth & 68 & 57.1 \\
Klebsiella spp. & 27 & 22.7 \\
Pseudomonas aeruginosa & 4 & 3.4 \\
Staphylococcus aureus & 16 & 13.4 \\
Escherichia coli & 2 & 1.7 \\
Proteus spp. & 1 & 0.8 \\
Pneumococci & 1 & 0.8 \\
Total & 119 & 100.0
\end{tabular}

countries continue to experience unacceptably high neonatal mortality.

There was a slight drop of admissions to the SCBU which was associated with a rise in mortality over the 3 years of the study. This finding can be explained by an increase in the number of preterm deliveries and its complications, the lack of availability of surfactants and an increase in congenital malformations all of which contribute to high mortality rates.

Our study revealed that gestational age (maturity) was the most important risk factor for neonatal mortality especially when accompanied by respiratory problems that needed ventilator support. About half of the neonatal deaths (52.3\%) were premature babies. Deaths among the extremely low birth weight and very low birth weight infants were largely attributed to respiratory insufficiency, necrotizing enterocolitis and infections. The majority of deaths were among neonates of birth weight $<1500$ mortality in babies with birth weight $>$ $1500 \mathrm{~g}-<2500 \mathrm{~g}$ (low birth weight). The mortality rates of very low and extremely low birth weight neonates are still high in most of the developed countries [8]. But the overall survival rate for very low birth weight newborns in Japan was around 90\% [14]. This improvement of survival of such infants reflects the improvement in medical care [15] and better knowledge of the pathophysiology of the neonate [13]. Very low birth weight is a preventable risk factor of neonatal death and decline in very low birth weight births will result in a decline of neonatal deaths in general [15]. In our study neonatal mortality decreased with increasing birth weight.

Surgical delivery is a well recognized method for reducing neonatal mortality [15]. Several studies indicate that caesarean section is the preferred mode of delivery for preterm infants with breech presentation [14]. Emergency g with $53 \%$ mortality as opposed to $16 \%$

\begin{tabular}{lccc}
\hline Table 4 Neonatal death rates in different countries & & \\
\hline References & No. of admissions & No. of deaths & $\%$ \\
Kenya [6] & 939 & 231 & 24.6 \\
Saudi Arabia [5] & 277 & 62 & 22.4 \\
Present study & 1176 & 260 & 22.1 \\
Zimbabwe [10] & 5305 & 1023 & 19.3 \\
Uganda [7]. & 1598 & 288 & 18.0 \\
Saudi Arabia [11]. & 969 & 169 & 17.4 \\
Taiwan [12] & 1083 & 153 & 14.1 \\
Australia [13] & 1776 & 111 & 6.3 \\
Canada [8] & 19265 & 795 & 4.1 \\
United States of America [9] & 47820 & 380 & 0.8 \\
\hline
\end{tabular}

caesarean section was the mode of delivery for $29.7 \%$ of the babies that died. These babies had probably suffered from fetal distress either in the womb or during delivery. They perhaps had not been monitored closely enough during labour, otherwise they might have been identified early enough and managed appropriately in order to avoid asphyxia and premature labour. Instrumental delivery was used in $2.5 \%$ of our neonates who died. More than half of our neonates were delivered by normal vaginal delivery, including preterm babies with breech presentation.

Male newborns had higher mortality than females but this was not statistically significant, which concurs with previous reports which indicate that sex is not a significant predictor of mortality $[10,16,17]$.

The majority of deaths $(63.1 \%)$ occurred during the early neonatal period. This indicates that the early neonatal period is more critical than the late neonatal period. These findings support the suggestion to establish and expand neonatal care services that are accessible and capable of providing immediate and appropriate specialized care to sick newborns, such as kangaroo care (skin to skin) [18]. At the same time, education and communication activities are needed to raise community awareness about the availability of neonatal services [19].

The factors significantly associated with neonatal mortality in our study in descending order of frequency were: prematurity with respiratory distress syndrome, congenital malformations, infections and perinatal asphyxia. These factors are similar to those observed in other neonatal units in the Saudi Arabia and Egypt but different from data from North American and European countries where the standard of health care is high $[12,20]$. Deaths among premature infants were largely attributed to respiratory insufficiency, so hyaline membrane disease is the main reason for such deaths. Little can be done to 
prevent prematurity, but morbidity and mortality associated with prematurity can be improved by: using drugs such as surfactants and nitric oxide, the prenatal use of dexamethasone and the use of sophisticated equipment like pulse oximeters, apnoea monitors, ventilators, oscillators and extracorporeal membrane oxygenation $[11,19,20]$. Exogenous surfactant was not always available during this study. Other well documented methods of improving the survival of premature infants include the use of antenatal corticosteroids and postnatal surfactants. These methods are still not widely used in our and other developing countries, especially surfactants which are quite expensive $[10,11,19,20]$.

Congenital malformations are an important contributor to neonatal mortality; the death rate was highest among those infants with cardiac lesions, followed by neural tube defects and other genetic anomalies. Some congenital anomalies are preventable $[15,20]$, such as neural tube defects that can be prevented by using folic acid during the preconception period [20]. Consanguineous marriage, extreme maternal age (over 40 years or very young mothers), socioenvironmental factors and chronic maternal disease such as diabetes are all factors associated with a higher incidence of congenital anomalies $[4,11,21]$. Although the consanguinity rate in this study was not known, the traditional pattern of consanguineous marriage in Arab and Islamic countries, including ours, may influence the frequency of autosomal recessive conditions and the incidence of congenital anomalies [11,22]. Many congenital anomalies can be treated surgically but the management of life-threatening anomalies suffers from many problems such as the lack of total parenteral nutrition, well trained neonatal surgeons and postoperative nursing care [8].

The presence of nasogastric and end tracheal tubes leaves babies highly susceptible to infections, especially among premature neonates. The predominant organisms causing neonatal sepsis in our study were Klebsiella spp., Staph. aureus, Pseudomonas spp.. These causative organisms for septicaemia are similar to those already reported in Saudi Arabia, but contrast with the dominant prevalence of group B streptococcus in the United States and United Kingdom [11]. High infection rates may be attributed to poor antiseptic measures, lack of qualified nursing staff and inadequate supply of appropriate antibiotics $[20,23]$. Physicians continue to use antibiotics inappropriately and excessively and neonatologists must promote the rational use of antibiotics [24].

The high rate of asphyxia may indicate deficiencies in the antenatal care and intrapartum management [11]. Therefore priority should be given to strengthening obstetric care in the community. Indeed, it indicates the need to examine the facilities for intrapartum monitoring, the mode of delivery and the availability of trained personnel.

To conclude, mortality in our SCBU is high and action is needed to identify and reduce risk factors for the neonatal mortality, with special emphasis on prematurity and its complications.

\section{Acknowledgement}

We would like to acknowledge each person who took part in this study for their effort: Dr Laila Sebai, consultant of clinical epidemiology for assisting with the statistical analysis of our data; Dr Khamila Elrafifi, consultant paediatrician and neonatologist; and Mr Khalid Ghorman and Miss Nairoz Bakir Abushhiwa both of them helped collect data. Finally, we thank Dr Abdelmaged Aborawi, consultant paediatrician, neonatologist and Head of the neonatal care unit for allowing us to use hospital data.

\section{References}

1. Avery ME, Taeusch W Jr., eds. Schafer's diseases of the newborn 5th ed. Philadelphia, WB Sauders, 1984.

2. World Health Organization 2006, Neonatal and Perinatal mortality, Country, Regional and Global Estimates, 1-6, 43-52.

3. Dekna $\mathrm{M}$ et al. Morbidity and mortality in Libyan neonates: a study from Aljala hospital Tripoli. Libya Jamahiriya Medical Journal, 2002, 2(1):48-53.

4. Behrman RE, Kliegman RM, Jenson HB. Nelson textbook of pediatrics, 17th ed., United States, Elsevier Science, 2004:519, 550-553,566-567,623-630.

5. Arafa MA, Alshehri MA. Predictors of neonatal mortality in the intensive care unit in Abha, Saudi Arabia. Saudi Medical Journal, 2003, 24:1374-1376.

6. Kasirye-Bainda E, Musoke FN. Neonatal morbidity and mortality at Kenyatta National hospital newborn unit. East Medical Journal, 1992, 69(7):360-364.
7. Mukasa GK. Morbidity and mortality in the Special Care Baby Unit of New Mulago Hospital, Kampala. Annals of Tropical Paediatrics, 1992, 12:289-295.

8. Sankaran $\mathrm{K}$ et al.; Canadian Neonatal Network. Variations in mortality rates among Canadian neonatal intensive care units. Canadian Medical Association Journal, 2002, 166:173-178.

9. Barton L, Hodgman JE. The contribution of the withholding or withdrawing care to newborn mortality. Pediatrics, 2005, 116(6):1487-1491.

10. Kambarami RA, Matibe P, Pirie D. Risk factors for neonatal mortality: Harare Central Hospital Neonatal Unit - Zimbabwe. Central African Journal of Medicine, 1999, 45(7):169-173.

11. Bassuni W et al. Neonatal deaths in the Asir Region of Saudi Arabia: Experience in a Referral Neonatal Intesive Care Unit. Annals of Saudi Medicine, 1997, 17:522-6.

12. Yau $\mathrm{KI}, \mathrm{Hsu} \mathrm{CH}$. Factors affecting the mortality of sick newborns admitted to intensive care units. Acta Paediatrica Taiwanica, 1999, 40(2):75-82. 
13. Wilkinson DJ et al. Death in the neonatal intensive care unit: changing pattern of end of life care over two decades. Archives of Disease in Childhood. Fetal and Neonatal Edition, 2006 94(1):F268-F271.

14. Kusuda $S$ et al. Morbidity and mortality of infants with very low birth weight in Japan: center variation. Pediatrics, 2006 , 118(4):e1130-e1138.

15. Ho NK. A study of 8 year neonatal deaths (1982-1989) of Toa Payoh Hospital. Singapore Medical Journal, 1991, 32:138-141.

16. Kambarami R, Chidede O, Chirisa M. Neonatal intensive care in a developing country: outcome and factors associated with mortality. Central African Journal of Medicine, 2000, 46:205-207.

17. Copper RL et al. A multicenter study of preterm birth weight and gestational age-specific neonatal mortality. American Journal of Obstetrics and Gynaecology, 1993, 168(1):78-84.

18. Campbell $\mathrm{O}$ et al. The Egypt national perinatal/neonatal mortality study 2000. Journal of Perinatology, 2004, 24:284-289.
19. Alobaidy.H et al. Perinatal mortality and morbidity at Alkhadra hospital Tripoli Libya. Jamahiriya Medical Journal, 2004, 3:48-51.

20. Abusrewil $S$ et al. Pattern of disease in neonates: a twelvemonth study. Jamahiriya Medical Journal, 2002, 2:44-48.

21. Kelnar CJ, Harvey D, Simpson C. The sick newborn baby, 3rd ed. London, Bailliere Tindall, 1995:197-248, 344-350.

22. Singh R, Al-Sudani O, Major congenital anomalies at birth in Benghazi, Libyan Arab Jamahiriya. Eastern Mediterranean Health Journal, 1995, 6(1):65-75.

23. Misallati A, el-Bargathy $S$, Shembesh N. Blood-culture-proven neonatal septicaemia: a review of 36 cases. Eastern Mediterranean Health Journal, 2000, 6:483-486.

24. Stoll BJ et al. Late onset sepsis in very low birth weight neonates: the experience of the NICHD neonatal research network. Pediatrics, 2002, 110(2):285-291.

\section{Causes of neonatal deaths}

Newborn, or neonatal, deaths account for $37 \%$ of all deaths among children under five. The majority of all neonatal deaths (75\%) occur during the first week of life, and of those deaths, between $25 \%$ to $45 \%$ occur within the first 24 hours.

The main causes of newborn deaths are prematurity and low-birth-weight (30\%), infections (25\%), asphyxia (lack of oxygen at birth) and birth trauma (23\%) thus accounting for nearly $80 \%$ of deaths in this age group.

WHO and its partners agree that a core principle underlying maternal, newborn and child health efforts is lifelong access to health care: a continuum of care for the mother starting from long before pregnancy (during childhood and adolescence) through pregnancy and childbirth. The continuum begins again with adequate newborn care for the new life. As appropriate, care can be delivered in the home and community, as well as health clinics and hospitals.

Source WHO Fact sheet, No.333

August 2009 